\title{
EFECTO DEL ALMACENAMIENTO A DIFERENTES TEMPERATURAS SOBRE EL DESARROLLO DE COLOR EXTERNO Y LA CALIDAD DE FRUTOS DE PIÑA CV. DORADA EXTRA DULCE
}

\begin{abstract}
Luis Ulloa*, Marco Vinicio Sáenz, ${ }^{1 / *}$, Johanny Castro*
Palabras clave: Piña; temperaturas de almacenamiento; cambio de color externo; clorofila; respiración; noclimatérico.

Keywords: Pineapple; storage temperature; external color change; chlorophyll; respiration; non-climacteric.
\end{abstract}

Recibido: 06/04/15

\begin{abstract}
RESUMEN
Con el objetivo de mejorar la calidad de la fruta, juzgada en el mercado como el grado de coloración amarilla de la cáscara, se llevaron a cabo 2 experimentos para evaluar el efecto de la temperatura de almacenamiento y transporte sobre el cambio en la coloración externa de frutos de piña Ananas comosus cv. "Dorada Extra Dulce". Las temperaturas fueron $7,5^{\circ} \mathrm{C} ; 10,0^{\circ} \mathrm{C}$; $12,5^{\circ} \mathrm{C}$ y $15,0^{\circ} \mathrm{C}$, y se midió su efecto sobre el cambio de color y el contenido de clorofila en la cáscara, la tasa respiratoria y parámetros de calidad externos e internos. La fruta fue almacenada bajo las diferentes temperaturas durante 21 días y posteriormente transferida a $18,0^{\circ} \mathrm{C}$ por 4 días adicionales. Al final del período de almacenamiento, las piñas almacenadas a $7,5^{\circ} \mathrm{C}$ mostraron el mayor contenido de clorofila $a$ (200 $\mu \mathrm{g} . \mathrm{g}^{-1}$ p.f) y el menor grado de color externo $(0,32-0,70)$; las que permanecieron a $15,0^{\circ} \mathrm{C}$ presentaron el menor contenido de clorofila $a\left(40 \mu \mathrm{g} . \mathrm{g}^{-1} \mathrm{p} . \mathrm{f}\right) \mathrm{y}$ el mayor grado de color $(4,42-5,25)$. La menor tasa de respiración a los 21 días se presentó en las piñas almacenadas a $7,5^{\circ} \mathrm{C}\left(6,4 \mathrm{ml} \mathrm{CO} / 2 \mathrm{~kg}^{*} \mathrm{~h}\right)$, mientras que frutas almacenadas a $15,0^{\circ} \mathrm{C}$ mostraron la mayor tasa respiratoria $\left(15,8 \mathrm{ml} \mathrm{CO} / / \mathrm{kg}^{*} \mathrm{~h}\right)$. Conforme aumentó la temperatura de almacenamiento se
\end{abstract}

1 Autor para correspondencia. Correo electrónico: marco.saenz@ucr.ac.cr
Aceptado: 12/06/15

\section{ABSTRACT}

Effect of storage at different temperatures on development of external color and quality of pineapple fruits cv. Gold Extra Sweet. In order to improve the quality of the fruit, judged at the market as the degree of yellowing of the peel, 2 experiments were performed to evaluate the effect of storage temperature on change in external color of pineapple (Ananas comosus) fruit cv. "Gold Extra Sweet". The temperatures evaluated were $7.5^{\circ} \mathrm{C} ; 10.0^{\circ} \mathrm{C}$; $12.5^{\circ} \mathrm{C}$ and $15.0^{\circ} \mathrm{C}$, and their effect on the color change and the chlorophyll content in the peel, respiration rate and parameters of external and internal quality were measured. The fruit was stored at different temperatures for 21 days and then transferred to $18.0^{\circ} \mathrm{C}$ during 4 additional days. At the end of the storage period, pineapples at $7.5^{\circ} \mathrm{C}$ showed the highest content of chlorophyll $a\left(200 \mu \mathrm{g} \cdot \mathrm{g}^{-1}\right.$ f.w $)$ and the lowest degree of external color $(0.32-0.70)$, fruits stored at $15.0^{\circ} \mathrm{C}$ had the lowest content of chlorophyll $a$ (40 $\mu \mathrm{g} \cdot \mathrm{g}^{-1}$ f.w) and the highest color grade (4.42-5.25). Pineapples stored at $7.5^{\circ} \mathrm{C}$ showed the lowest respiration rate at 21 days $\left(6.4 \mathrm{ml} \mathrm{CO} / \mathrm{kg}^{*} \mathrm{~h}\right)$, while fruit stored at $15.0^{\circ} \mathrm{C}$ had the highest respiration rate (15.8 $\left.\mathrm{ml} \mathrm{CO}{ }_{2} / \mathrm{kg}^{*} \mathrm{~h}\right)$. As the storage temperature increased the weight loss and mold growth in

\footnotetext{
* Laboratorio de Tecnología de Poscosecha, Centro de Investigaciones Agronómicas, Universidad de Costa Rica. San José, Costa Rica.
} 
incrementó la pérdida de peso y el desarrollo de mohos en el pedúnculo. Al final del período de almacenamiento a $18,0^{\circ} \mathrm{C}$, la fruta previamente almacenada a $7,5^{\circ} \mathrm{C}$ tuvo escaso avance de color, mientras que las provenientes de $15,0^{\circ} \mathrm{C}$ tuvieron un mayor desarrollo de color amarillo. Los datos obtenidos sugieren que la temperatura de almacenamiento afecta la degradación de clorofila y el desarrollo de color amarillo en la cáscara de los frutos, lo cual podría ser de utilidad en el manejo comercial de la piña.

\section{INTRODUCCIÓN}

El desarrollo de color amarillo en la cáscara de los frutos de piña, es un atributo de calidad deseable en los mercados internacionales (Garita 2014). Este proceso ocurre durante la maduración del fruto y a través de la degradación de la clorofila, cuando el cloroplasto se transforma en cromoplasto (Hassan y Othman 2011).

Una vez que la clorofila se ha degradado, se da la expresión de otros pigmentos como carotenoides y antocianinas, que se encontraban enmascarados por la acción de la clorofila (Symons et ál. 2012). En este proceso, están involucradas enzimas como las clorofilasas, magnesio dequelatasas y una tercera conocida como Feoforbido $a$ oxígenasa (Wang et ál. 2005).

En la vida poscosecha de las frutas, la temperatura es considerada como el factor ambiental más importante para mantener la calidad de las mismas, debido al efecto que ejerce en el control de procesos biológicos y enzimáticos (Hong et ál. 2013). Diversos autores han hecho referencia al efecto del uso de diferentes temperaturas de almacenamiento, en el desarrollo de color en frutos no climatéricos como las mandarinas (Tietel et ál. 2012), granada real (Fawole y Opora 2013) y fresa (Mazur et ál. 2014). Abdullah et ál. (2002) y Liu y Liu (2014), almacenaron frutos de piña a diferentes temperaturas y observó que el mayor desarrollo de color amarillo se presentó the peduncle increased. At the end of storage at $18.0^{\circ} \mathrm{C}$, the color advance in the fruits previously stored at $7.5^{\circ} \mathrm{C}$ was the lowest, while the highest external yellow color developed on the fruits previously kept at $15.0^{\circ} \mathrm{C}$. The results suggest that the storage temperature affects chlorophyll degradation and development of external yellow color, which could be useful in the commercial management of pineapple fruit.

en aquellas que permanecieron en las temperaturas más elevadas.

Para el cv. Dorada Extra Dulce, fruta que presenta gran aceptación en el mercado internacional (Garita 2014), existe poca información sobre el cambio de color durante la vida poscosecha. Solano (2015) reportó que, luego de almacenar frutos de piña de esta variedad por 21 días a $7,0^{\circ} \mathrm{C}$ (condiciones comerciales), se observó escaso desarrollo de color amarillo en la cáscara de los frutos.

Ya que la información disponible indica que entre menor sea la temperatura a la cual se almacenan los frutos de piña, menor es el desarrollo de color amarillo que presentan en el mercado internacional, donde se prefiere piñas con mayor color amarillo. Por lo que se planteó la presente investigación, con el objetivo de evaluar el efecto de diferentes temperaturas de almacenamiento sobre la degradación de clorofila y cambio de color en la cáscara, y calidad general de frutos de piña cv. Dorada Extra Dulce.

\section{MATERIALES Y MÉTODOS}

\section{Material vegetal y diseño experimental}

Se desarrollaron 2 experimentos en el 2014 realizados en julio y en octubre respectivamente, con el fin de evaluar el efecto de diferentes 
temperaturas de almacenamiento, sobre el cambio de color, en fruta producida bajo diferentes condiciones climáticas. Se utilizaron frutos de piña cv. Dorada Extra Dulce, provenientes de una finca dedicada a la producción comercial, localizada en Pital de San Carlos, provincia de Alajuela, Costa Rica.

Para ambos experimentos se seleccionaron frutos destinados para exportación que recibieron el proceso poscosecha completo, el cual incluyó la desinfección con cloro (120-150 mg..$^{-1}$ de cloro libre), aplicación de cera a base de ácidos grasos, y fungicida en el pedúnculo.

Se seleccionó fruta en el grado 0 según la escala comercial de color (Figura 1), y que corresponde a un 100 de color verde en la cáscara. Las frutas se empacaron en cajas de cartón corrugado, colocándose 6 frutas por caja; posteriormente, se transportaron a temperatura ambiente hasta el Laboratorio de Tecnología Poscosecha, del Centro de Investigaciones Agronómicas, de la
Universidad de Costa Rica. El tiempo de transporte fue de aproximadamente 4 horas.

Las frutas se dividieron en 4 grupos, y se almacenaron a $7,5^{\circ} \mathrm{C}$ (Tratamiento control); $10,0^{\circ} \mathrm{C} ; 12,5^{\circ} \mathrm{C}$ y $15,0^{\circ} \mathrm{C}$ respectivamente durante 21 días, más 4 días a $18,0^{\circ} \mathrm{C}$, para simular las condiciones de anaquel $(21+4)$.

En ambos ensayos, a los 0,21 y 21+4 días de almacenamiento (dda), se evaluó el cambio de color en la cáscara de la fruta a través de la escala de color comercial; asimismo, se determinó la calidad de las frutas mediante la pérdida de peso, deshidratación parcial de las aristas (DPA) y cobertura de moho en el pedúnculo. A los 0 y 21+4 dda, se evaluó la firmeza y el porcentaje de translucidez en la pulpa, el porcentaje sólidos solubles y la acidez titulable. Solamente, en el segundo ensayo a los 0,21 y $21+4$ dda se determinó la tasa respiratoria, y a los 0 y $21+4$ dda se obtuvo el contenido de clorofila en la cáscara.
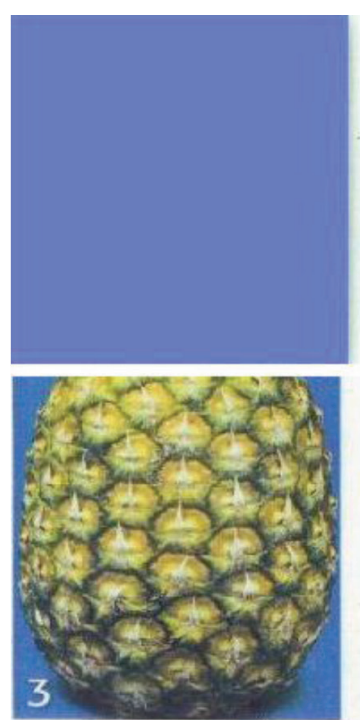
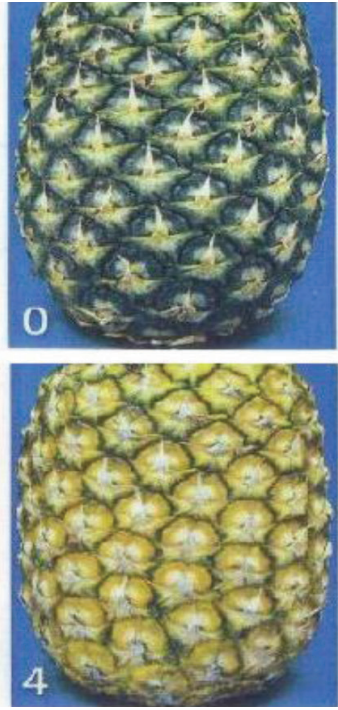
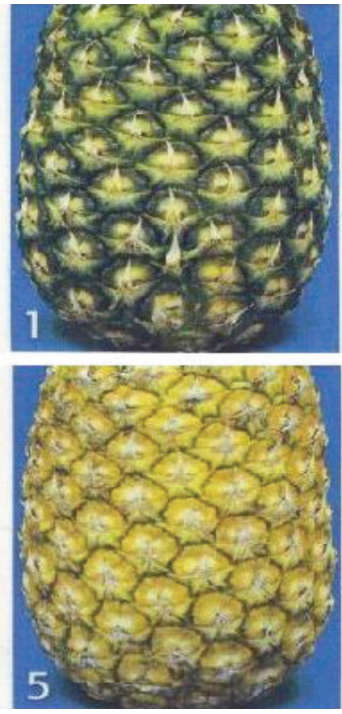
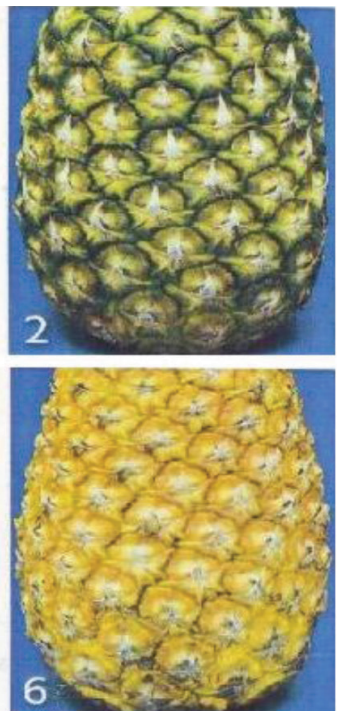

Fig. 1. Escala de color externo de frutos de piña, basada en la descripción realizada por Paull y Chen (2003).

\section{Diseño experimental}

El diseño experimental utilizado en ambos ensayos fue un irrestricto al azar con 4 tratamientos (temperaturas de almacenamiento). Se realizó un análisis de varianza con una comparación de promedios mediante la prueba de Tukey $(\mathrm{p}=0,05)$. Para las variables de contenido de clorofila y tasa de respiración se trabajó con un total de 6 
repeticiones, para las demás variables se utilizaron 5 repeticiones y la unidad experimental estuvo constituida por una caja con 6 frutas (unidad comercial).

\section{Porcentaje de cambio de color externo y contenido de clorofila}

Se evaluó el color externo de los frutos de piña, mediante la escala comercial (Figura 1), en la cual el grado 0 corresponde a un $100 \%$ de color verde y el grado 6 a un $100 \%$ de color amarillo. Esta variable se reportó como porcentaje de cambio de color externo, con respecto al color inicial de cada fruta.

La extracción de la clorofila se realizó con acetona, de acuerdo con los métodos descritos por Dere et ál. (1998) y Roberts (2009). La absorbancia se determinó en un espectrofotómetro Thermo scientific, modelo Genesys 10s. El contenido de clorofila (chl) $a$ y $b$ se expresó como microgramos por gramo de peso fresco ( $\mu$ g.g $\mathrm{g}^{-1}$ p.f.).

\section{Tasa de respiración}

Inicialmente se pesó la fruta y se colocó en recipientes herméticos de $30 \mathrm{~L}$, durante 4 horas. Transcurrido este tiempo, se obtuvo el porcentaje de dióxido de carbono $\left(\mathrm{CO}_{2}\right)$, con un analizador de gases Brigde Analyzer, MAP Headspace $\mathrm{O}_{2} / \mathrm{CO}_{2}$, Modelo 9001. Con los datos de $\mathrm{CO}_{2}$ para cada fruta, se calculó la tasa respiratoria en $\mathrm{ml} \mathrm{CO}_{2} / \mathrm{kg}^{*}$ h según lo descrito por Umaña et ál. (2011).

\section{Parámetros de calidad de la fruta}

Se obtuvo el peso de los frutos en cada fecha de evaluación y se reportó como pérdida de peso con respecto al valor inicial. Se determinó la incidencia y severidad de DPA o "pitting", con base en la evaluación de 10 frutículos en cada fruta, en donde se evaluó la presencia o no de hundimientos en los bordes de los mismos (incidencia), y cuántos de estos afectaban el frutículo en más de un $25 \%$ de su superficie (severidad). Además, se determinó el porcentaje de área del pedúnculo cubierta por moho.

\section{Calidad interna de la fruta}

Se determinó la translucidez de la pulpa, que se reportó como porcentaje de avance desde la base hacia la zona apical. La Firmeza de la pulpa se evaluó en 2 puntos equidistantes en la zona media de la pulpa y se expresó en Newton $(\mathrm{N})$, para lo cual se utilizó un penetrómetro QA supplies modelo FT011, con una punta convexa de $7,93 \mathrm{~mm}$.

Para determinar el porcentaje de sólidos solubles y acidez titulable, se extrajo una sección de pulpa de aproximadamente $3 \mathrm{~cm}$ de ancho, a través de un corte longitudinal, del cual se obtuvo el jugo mediante un extractor manual, y se filtró con 4 capas de gasa. A partir de este jugo, se determinó el porcentaje de sólidos solubles, con un refractómetro digital Atago PAL-1. La acidez titulable se obtuvo mediante una titulación con una solución de $\mathrm{NaOH} 0,1 \mathrm{~N}$, y se utilizó como indicador fenolftaleína al $1 \%$. El porcentaje de acidez titulable se calculó como porcentaje de ácido cítrico.

\section{RESULTADOS Y DISCUSIÓN}

\section{Porcentaje de cambio de color externo y contenido de clorofila}

En el Cuadro 1, se muestran los resultados de color externo y porcentaje de avance de color en la cáscara de frutos de piña cv. Dorada Extra Dulce, almacenados a diferentes temperaturas durante 21 días, más 4 días a $18,0^{\circ} \mathrm{C}$. Para las evaluaciones realizadas tanto a los 21 , como a los 21+4 dda de ambos ensayos, se mostraron diferencias significativas entre los tratamientos en el porcentaje de cambio de color $(\mathrm{p}<0,0001)$. Las frutas del tratamiento control fueron las que obtuvieron el menor porcentaje, mientras que el mayor cambio de color externo lo presentaron las frutas que permanecieron almacenadas a $15,0^{\circ} \mathrm{C}$.

Abdullah et ál. (2002), almacenaron frutos de piña cv. Gandul a diferentes temperaturas y observaron que, luego de 5 semanas de almacenamiento, aquellos que se mantuvieron en $5^{\circ} \mathrm{C}$ y $10^{\circ} \mathrm{C}$, presentaron escaso desarrollo de 
Cuadro 1. Color y porcentaje de cambio de color externo de la cáscara de frutos de piña almacenados a diferentes temperaturas.

\begin{tabular}{|c|c|c|c|c|c|}
\hline \multirow{3}{*}{ Tratamiento } & \multicolumn{3}{|c|}{ Promedio de color externo } & \multicolumn{2}{|c|}{$\%$ Cambio color externo } \\
\hline & \multicolumn{3}{|c|}{ Días de almacenamiento } & \multicolumn{2}{|c|}{ Días de almacenamiento } \\
\hline & 0 & 21 & $21+4$ & 21 & $21+4$ \\
\hline \multicolumn{6}{|c|}{ Experimento 1} \\
\hline $7,5^{\circ} \mathrm{C}$ & $0,28 \pm 0,1^{*}$ & $0,28 \pm 0,11$ & $0,32 \pm 0,17$ & $3 \mathrm{a}^{\dagger}$ & $13 \mathrm{a}$ \\
\hline $10,0^{\circ} \mathrm{C}$ & $0,22 \pm 0,1$ & $0,45 \pm 0,19$ & $0,93 \pm 0,26$ & $88 \mathrm{a}$ & $275 \mathrm{~b}$ \\
\hline $12,5^{\circ} \mathrm{C}$ & $0,20 \pm 0,1$ & $1,00 \pm 0,45$ & $2,77 \pm 1,27$ & $321 \mathrm{~b}$ & $1040 \mathrm{c}$ \\
\hline $15,0^{\circ} \mathrm{C}$ & $0,21 \pm 0,1$ & $3,29 \pm 0,90$ & $5,25 \pm 0,65$ & $1254 \mathrm{c}$ & $2031 \mathrm{~d}$ \\
\hline \multicolumn{6}{|c|}{ Experimento 2} \\
\hline $7,5^{\circ} \mathrm{C}$ & $0,21 \pm 0,19$ & $0,52 \pm 0,36$ & $0,70 \pm 0,38$ & $100 \mathrm{a}$ & $156 \mathrm{a}$ \\
\hline $10,0^{\circ} \mathrm{C}$ & $0,14 \pm 0,16$ & $0,49 \pm 0,48$ & $1,24 \pm 0,41$ & $109 \mathrm{a}$ & $423 \mathrm{~b}$ \\
\hline $12,5^{\circ} \mathrm{C}$ & $0,33 \pm 0,26$ & $1,96 \pm 0,56$ & $2,07 \pm 0,51$ & $528 \mathrm{~b}$ & $569 \mathrm{~b}$ \\
\hline $15,0^{\circ} \mathrm{C}$ & $0,23 \pm 0,19$ & $2,56 \pm 0,50$ & $4,42 \pm 1,25$ & $822 \mathrm{c}$ & $1542 \mathrm{c}$ \\
\hline
\end{tabular}

*Valores son el promedio de la medición de 6 frutas por repetición. \pm Indica la desviación estándar.

Letras distintas indican diferencias significativas según prueba de Tukey $(\mathrm{p}=0,05)$.

color amarillo en la cáscara, mientras que los provenientes de temperaturas de $15^{\circ} \mathrm{C}$ y $20^{\circ} \mathrm{C}$, presentaron el mayor avance de color. Asimismo, Liu y Liu (2014) almacenaron frutos de piña a diferentes temperaturas durante 36 horas y encontraron que, el mayor desarrollo de color amarillo, se presentó en aquellas que se almacenaron a temperaturas elevadas. Los datos de los autores anteriores, quienes trabajaron con variedades distintas a la Dorada Extra Dulce, concuerdan con lo encontrado en este trabajo, e indican que entre mayor sea la temperatura a la cual se almacenen los frutos de piña, mayor será el desarrollo de color amarillo que estas presenten.

En la Figura 2, se muestra el contenido de clorofila $a$ y $b\left(\mu \mathrm{g} \cdot \mathrm{g}^{-1}\right.$ p.f.), con base en el peso fresco en la cáscara de frutos de piña. Al finalizar los 21+4 dda, se evidenció una disminución en el contenido de clorofila, en aquellos frutos que permanecieron en las mayores temperaturas de almacenamiento $\left(12,5^{\circ} \mathrm{C}\right.$ y $\left.15,0^{\circ} \mathrm{C}\right)$. Los frutos almacenados a $7,5^{\circ} \mathrm{C}$ durante 21 días, fueron los que presentaron el mayor contenido de clorofila $a$ y $b, \sin$ mostrar diferencias significativas con respecto a la evaluación inicial. Mientras que los frutos que estuvieron almacenados a $15,0^{\circ} \mathrm{C}$, mostraron el menor contenido de clorofila $a$ y $b, \mathrm{y}$ fueron estadísticamente diferentes al tratamiento control de $7,5^{\circ} \mathrm{C}(\mathrm{p}<0,0001)$.

En este trabajo se evidenció que, el cambio de color externo y la degradación de la clorofila, se vieron afectados por el aumento en la temperatura de almacenamiento. En el tratamiento control, se presentaron contenidos de clorofila similares a la evaluación inicial, con escaso desarrollo de color amarillo, mientras que en las temperaturas de $12,5^{\circ} \mathrm{C}$ y $15,0^{\circ} \mathrm{C}$, ocurrió lo contrario. Brat et ál. (2004), mencionan que en frutos de piña, el color amarillo externo característico de la maduración, se debe a la degradación de la clorofila y a la expresión de carotenoides; tal y como se observó en la presente investigación, en donde los frutos que presentaron un mayor desarrollo de color amarillo al final del período de almacenamiento, mostraron menor contenido de clorofila. 

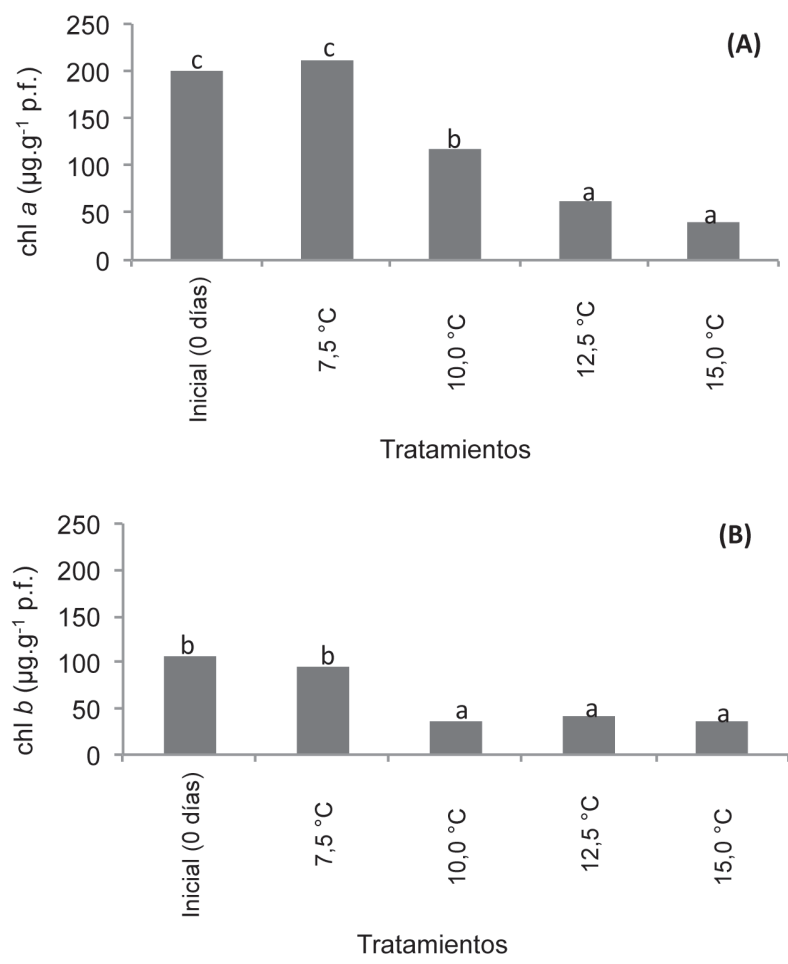

Fig. 2. Contenido promedio de clorofila en cáscaras de frutos de piña almacenados a diferentes temperaturas. Letras distintas indican diferencias significativa según prueba de Tukey $(\mathrm{p}=0,05)$. Clorofila a (A) Clorofila b (B).

\section{Tasa de respiración}

En la Figura 3, se presenta la tasa de respiración de los frutos de piña, la cual aumentó conforme se incrementó la temperatura de almacenamiento. A los 21 dda, todos los tratamientos evidenciaron diferencias significativas $(\mathrm{p}<0,0001)$; aunque el tratamiento control fue el que presentó la menor tasa de respiración con $6 \mathrm{ml} \mathrm{CO} / 2 \mathrm{~kg}^{*} \mathrm{~h}$, mientras que la mayor tasa respiratoria se obtuvo en los frutos almacenados a $15,0^{\circ} \mathrm{C}$, con valores de $15 \mathrm{ml} \mathrm{CO} / / \mathrm{kg}^{*} \mathrm{~h}$. A los $21+4$ dda, no se mostraron diferencias significativas entre tratamientos.

La temperatura de almacenamiento influyó en la tasa de respiración de los frutos de piña pues, en el tratamiento control no se observaron cambios entre la evaluación inicial y a los 21 dda. Mientras que para la misma fecha de evaluación, los frutos almacenados a $15,0^{\circ} \mathrm{C}$, presentaron el mayor incremento en la tasa respiratoria con respecto a los $0 \mathrm{dda}$, con un cambio de $9,9 \mathrm{ml} \mathrm{CO}_{2} /$ $\mathrm{kg} * \mathrm{~h}$. $\mathrm{Al}$ pasar a $18,0^{\circ} \mathrm{C}$, se presentó un incremento en la tasa de respiración de todos los frutos; aquellos provenientes de las temperaturas de almacenamiento de $7,5^{\circ} \mathrm{C}$ y $10,0^{\circ} \mathrm{C}$ presentaron el mayor incremento en esta variable, con una diferencia de $11 \mathrm{ml} \mathrm{CO} / / \mathrm{kg}^{*}$ h entre ambas fechas de evaluación. El menor cambio en la tasa de respiración se presentó en los frutos que provenían de la temperatura de almacenamiento $15,0^{\circ} \mathrm{C}$, pues mostraron un cambio de $1,7 \mathrm{ml} \mathrm{CO} / \mathrm{kg} * \mathrm{~h}$. 


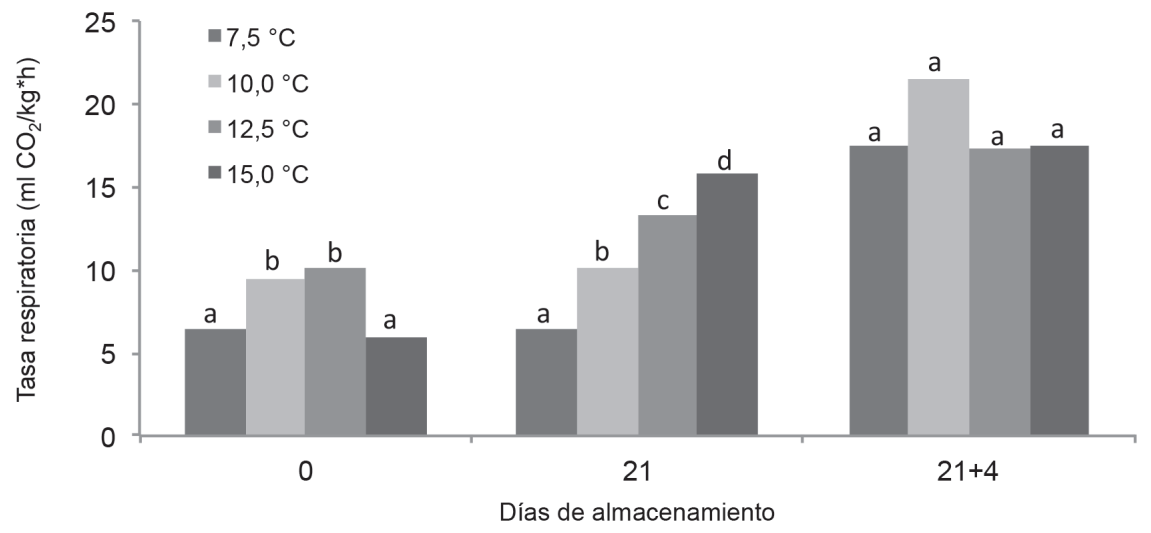

Fig. 3. Tasa respiratoria promedio $\left(\mathrm{ml} \mathrm{CO} 2 / \mathrm{kg}^{*} \mathrm{~h}\right)$ de frutos de piña almacenadas a diferentes temperaturas. Letras distintas indican la diferencias significativas según prueba de Tukey $(\mathrm{p}=0,05)$.

El incremento en la tasa respiratoria, como respuesta al aumento en la temperatura de almacenamiento, se da como consecuencia de una disminución en la energía de activación necesaria para llevar a cabo las diferentes reacciones involucradas en este proceso (Kays 1997, Fonseca et ál. 2002).

\section{Calidad externa de la fruta}

En la Figura 4, se muestra el porcentaje de pérdida de peso de los frutos de piña. Para el experimento 1 a los 21 dda (Figura 4A), el menor porcentaje de pérdida de peso se evidenció en los frutos que se almacenaron a $7,5^{\circ} \mathrm{C}$, mientras que las piñas almacenadas a $10,0^{\circ} \mathrm{C}$, fueron las que presentaron la mayor de pérdida de peso con un $12 \%$ ( $\mathrm{p}<0,0001)$. El mismo comportamiento se obtuvo a los $21+4$ dda, con diferencias estadísticas entre tratamientos $(\mathrm{p}<0,0001)$, y con un máximo de $13,1 \%$ en la temperatura de $10,0^{\circ} \mathrm{C}$.

El porcentaje de pérdida de peso a los 21 dda, del experimento 2 (Figura 4B), presentó diferencias significativas entre tratamientos $(\mathrm{p}<0,0001)$. La menor pérdida de peso se presentó en las piñas almacenadas a $7,5^{\circ} \mathrm{C}$, mientras que los frutos almacenados a $12,5^{\circ} \mathrm{C}$ presentaron el mayor porcentaje de pérdida de peso, el cual fue de $5 \%$. Este mismo comportamiento se obtuvo a los $21+4$ dda, con un máximo de 5,7\% en el tratamiento de $12,5^{\circ} \mathrm{C}$, con diferencias significativas entre tratamientos $(\mathrm{p}<0,0001)$.

El aumento en la pérdida de peso, conforme se incrementó la temperatura de almacenamiento, pudo estar influenciado por una mayor pérdida de humedad, debido a una mayor transpiración de los frutos y a una mayor tasa respiratoria, respuesta que ha sido documentada por diversos autores en piña y en otros tipos de frutos (Kader 2002, Hassan y Othman 2011).

La cámara que se encontraba a $10,0^{\circ} \mathrm{C}$, en el ensayo 1, presentó una humedad relativa menor a $80 \%$, que es un valor más bajo que el óptimo para el almacenamiento poscosecha de frutos de piña, el cual debe estar entre 90\% y 95\% (Paull y Chen 2003). Hassan y Othman (2011) mencionan que, bajas humedades relativas durante el almacenamiento de frutos de piña, favorecen la pérdida de peso, lo cual explicaría el alto porcentaje de pérdida de peso que soportaron los frutos que permanecieron en esta cámara. 

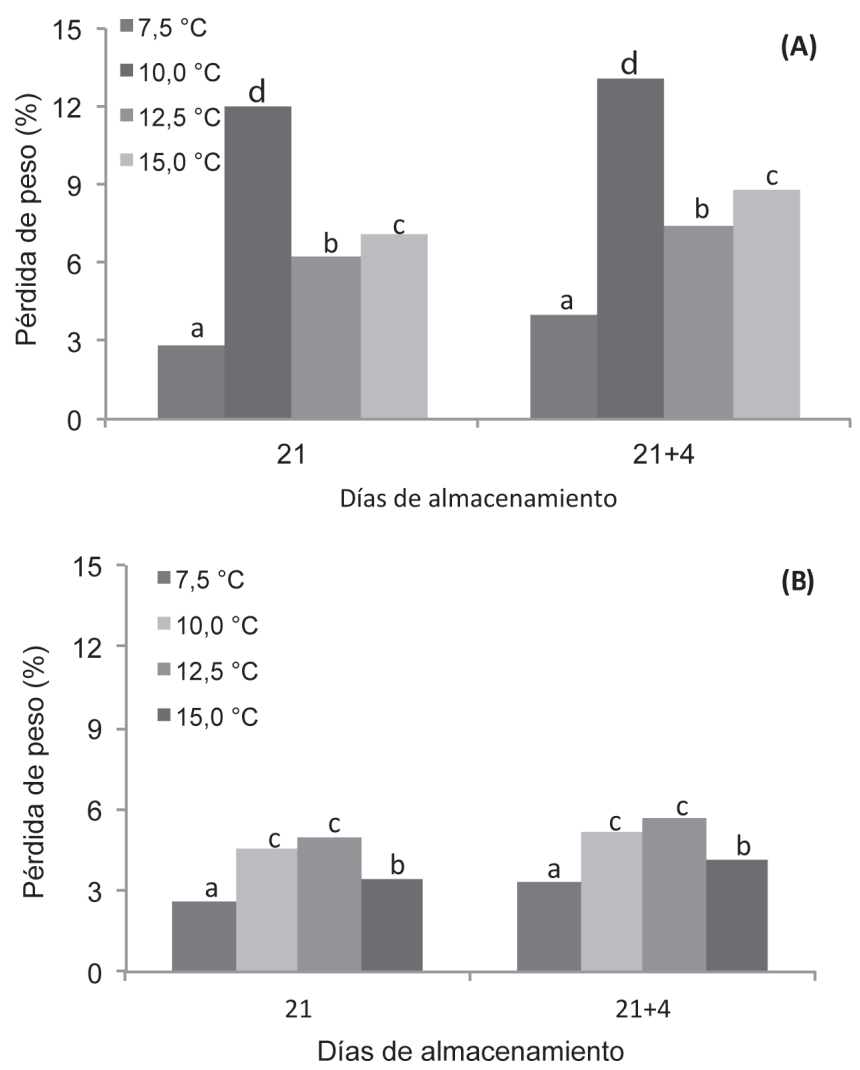

Fig. 4. Pérdida de peso (\%) de frutos de piña almacenados a diferentes temperaturas. Experimento 1 (A) y Experimento 2 (B). Letras distintas indican diferencias significativas según prueba de Tukey $(\mathrm{p}=0,05)$.

En ambos experimentos, en la evaluación inicial, no se presentaron diferencias significativas en la incidencia y severidad de DPA en la cáscara del fruto (Cuadro 2). A los 21 días de almacenamiento, solamente el tratamiento de $10,0^{\circ} \mathrm{C}$ del experimento 1 , presentó diferencias significativas en la incidencia de DPA con respecto al control, lo cual pudo estar influenciado por la humedad relativa a la que fue sometida la fruta durante su almacenamiento. A los $21+4 \mathrm{dda}$, se obtuvo valores de incidencia entre $31 \%$ y $78 \%$ en el experimento 1 , y entre $39 \%$ y $44 \%$ en el experimento 2 , sin diferencias estadísticas entre tratamientos. Tanto a los 21, como a los $21+4$ dda, solamente los frutos almacenados a $10,0^{\circ} \mathrm{C}$, presentaron una mayor severidad de DPA, con respecto al control.

$\mathrm{El}$ incremento en incidencia y severidad de DPA, tanto a los 21 dda, como a los $21+4$ dda, con respecto a la evaluación inicial en todos los tratamientos, muestra el efecto negativo de la pérdida de peso sobre la calidad externa de los frutos, situación también informada por Solano (2015) en el cv. Dorada Extra Dulce.

Para ambos experimentos se evidenció que, conforme se aumentó la temperatura de almacenamiento, se incrementó el porcentaje de área cubierta por mohos (Cuadro 2). En ambos ensayos, a los 21 dda, el menor desarrollo de moho se presentó en los frutos almacenados a 
Cuadro 2. Incidencia y Severidad de DPA y porcentaje de área del pedúnculo afectada por mohos en frutos de piña almacenados a diferentes temperaturas.

\begin{tabular}{|c|c|c|c|c|c|c|c|c|c|}
\hline \multirow{3}{*}{ Tratamiento } & \multicolumn{3}{|c|}{ Incidencia DPA (\%) } & \multicolumn{3}{|c|}{ Severidad DPA (\%) } & \multicolumn{3}{|c|}{ Mohos pedúnculo (\%) } \\
\hline & \multicolumn{3}{|c|}{ Días de almacenamiento } & \multicolumn{3}{|c|}{ Días de almacenamiento } & \multicolumn{3}{|c|}{ Días de almacenamiento } \\
\hline & 0 & 21 & $21+4$ & 0 & 21 & $21+4$ & 0 & 21 & $21+4$ \\
\hline \multicolumn{10}{|c|}{ Experimento 1} \\
\hline $7,5^{\circ} \mathrm{C}$ & $8,0 \mathrm{a}^{*}$ & $25,0 \mathrm{a}$ & $31,0 \mathrm{a}$ & $0,0 \mathrm{a}$ & $1,0 \mathrm{a}$ & $5,0 \mathrm{a}$ & $0,0 \mathrm{a}$ & $2,0 \mathrm{a}$ & $26,0 \mathrm{a}$ \\
\hline $10,0^{\circ} \mathrm{C}$ & $7,0 \mathrm{a}$ & $60,0 \mathrm{~b}$ & $78,0 \mathrm{a}$ & $0,0 \mathrm{a}$ & $18,0 \mathrm{~b}$ & $38,0 \mathrm{~b}$ & $0,0 \mathrm{a}$ & $5,0 \mathrm{a}$ & $90,0 \mathrm{~b}$ \\
\hline $12,5^{\circ} \mathrm{C}$ & $9,0 \mathrm{a}$ & $38,0 \mathrm{a}$ & $46,0 \mathrm{a}$ & $0,0 \mathrm{a}$ & $9,0 \mathrm{~b}$ & $7,0 \mathrm{a}$ & $0,0 \mathrm{a}$ & $90,0 \mathrm{~b}$ & $100,0 \mathrm{c}$ \\
\hline $15,0^{\circ} \mathrm{C}$ & $5,0 \mathrm{a}$ & $43,0 \mathrm{a}$ & $42,0 \mathrm{a}$ & $0,0 \mathrm{a}$ & $13,0 \mathrm{~b}$ & $8,0 \mathrm{a}$ & $0,0 \mathrm{a}$ & $100,0 \mathrm{~b}$ & $100,0 \mathrm{c}$ \\
\hline \multicolumn{10}{|c|}{ Experimento 2} \\
\hline $7,5^{\circ} \mathrm{C}$ & $16,0 \mathrm{a}$ & $29,0 \mathrm{a}$ & $40,0 \mathrm{a}$ & $0,0 \mathrm{a}$ & $4,0 \mathrm{a}$ & $5,0 \mathrm{a}$ & $0,0 \mathrm{a}$ & $0,0 \mathrm{a}$ & $4,0 \mathrm{a}$ \\
\hline $10,0^{\circ} \mathrm{C}$ & $11,0 \mathrm{a}$ & $31,0 \mathrm{a}$ & $44,0 \mathrm{a}$ & $1,0 \mathrm{a}$ & $5,0 \mathrm{a}$ & $8,0 \mathrm{ab}$ & $0,0 \mathrm{a}$ & $5,0 \mathrm{a}$ & $48,0 \mathrm{~b}$ \\
\hline $12,5^{\circ} \mathrm{C}$ & $9,0 \mathrm{a}$ & $23,0 \mathrm{a}$ & $39,0 \mathrm{a}$ & $0,0 \mathrm{a}$ & $4,0 \mathrm{a}$ & $12,0 \mathrm{~b}$ & $0,0 \mathrm{a}$ & $24,0 \mathrm{~b}$ & $53,0 \mathrm{~b}$ \\
\hline $15,0^{\circ} \mathrm{C}$ & $18,0 \mathrm{a}$ & $31,0 \mathrm{a}$ & $42,0 \mathrm{a}$ & $1,0 \mathrm{a}$ & $8,0 \mathrm{a}$ & $10,0 \mathrm{ab}$ & $0,0 \mathrm{a}$ & $82,0 \mathrm{c}$ & $94,0 \mathrm{c}$ \\
\hline
\end{tabular}

* Medias dentro de la misma columna con distinta letra, para cada experimento, difieren significativamente según la prueba de Tukey $(\mathrm{p}=0,05)$.

$7,5^{\circ} \mathrm{C}$ y $10,0^{\circ} \mathrm{C}$, obteniéndose como máximo un $5 \%$ de cobertura del pedúnculo, mientras que en los frutos almacenados a $12,5^{\circ} \mathrm{C}$ y $15,0^{\circ} \mathrm{C}$, se obtuvo entre $24 \%$ y $100 \%$ de crecimiento de moho en este tejido. A los $21+4$ dda, los porcentajes más bajos de moho en ambos experimentos $(\mathrm{p}<0,0001)$, se obtuvieron en el tratamiento control, donde se obtuvo como máximo un $26 \%$ en el primer ensayo.

Los resultados obtenidos indican que, para ambos experimentos, el desarrollo de mohos se vio favorecido por el incremento en las temperaturas de almacenamiento. Agrios (2005), menciona que entre más cercana la temperatura a la que se somete un patógeno, con respecto a su temperatura óptima de crecimiento, se favorece la formación y liberación de esporas del mismo, al completar más ciclos de desarrollo en un tiempo menor. Para patógenos como Penicillium sp., y Fusarium sp., géneros comúnmente relacionados con el desarrollo de mohos en el pedúnculo de frutos de piña (Garita 2014), las temperaturas óptimas de crecimiento se encuentran cercanas a los $20^{\circ} \mathrm{C}$ (Baert et ál. 2007, Gougouli y Koutsounamis 2010), lo cual explicaría el alto desarrollo de moho obtenido en los frutos almacenados a $15,0^{\circ} \mathrm{C}$.

\section{Calidad interna de la fruta}

En el Cuadro 3, se muestran las variables de calidad interna de los frutos de piña. En cuanto a la translucidez de la pulpa, que se presenta cuando los espacios intercelulares se llenan de líquido, relacionado a una mayor permeabilidad de las membranas y cambios en el potencial osmótico de las células (Paull y Chen 2003), no se observó una relación entre esta variable y las temperaturas de almacenamiento. Solamente en el 
Cuadro 3. Porcentaje de avance translucidez, firmeza (N), sólidos solubles (\%) y porcentaje acidez titulable en la pulpa de frutos de piña almacenados a diferentes temperaturas.

\begin{tabular}{ccccc}
\hline \multirow{2}{*}{ Tratamiento } & \multicolumn{4}{c}{ Calidad interna de la fruta } \\
\cline { 2 - 5 } & \% Avance translucidez & Firmeza (N) & Sólidos solubles (\%) & \% Acidez titulable \\
\hline Experimento 1 & & & & \\
Inicial (0 días) & 69,6 & 14,5 & 14,4 & $\mathrm{ND}^{\dagger}$ \\
$7,5^{\circ} \mathrm{C}$ & $28,8 \mathrm{a}^{*}$ & $15,2 \mathrm{~b}$ & $13,0 \mathrm{ab}$ & $\mathrm{ND}$ \\
$10,0^{\circ} \mathrm{C}$ & $39,4 \mathrm{a}$ & $14,9 \mathrm{~b}$ & $14,0 \mathrm{~b}$ & $\mathrm{ND}$ \\
$12,5^{\circ} \mathrm{C}$ & $14,4 \mathrm{a}$ & $10,8 \mathrm{a}$ & $13,1 \mathrm{ab}$ & $\mathrm{ND}$ \\
$15,0^{\circ} \mathrm{C}$ & $24,5 \mathrm{a}$ & $10,9 \mathrm{a}$ & $12,2 \mathrm{a}$ & $\mathrm{ND}$ \\
\hline Experimento & & & & \\
Inicial $(0$ días) & 9,2 & 19,8 & 14,5 & 0,5 \\
$7,5^{\circ} \mathrm{C}$ & $20,6 \mathrm{ab}$ & $12,5 \mathrm{~b}$ & $12,6 \mathrm{ab}$ & $0,70 \mathrm{ab}$ \\
$10,0^{\circ} \mathrm{C}$ & $25,6 \mathrm{~b}$ & $11,7 \mathrm{ab}$ & $13,6 \mathrm{~b}$ & $0,78 \mathrm{~b}$ \\
$12,5^{\circ} \mathrm{C}$ & $15,1 \mathrm{a}$ & $11,4 \mathrm{ab}$ & $11,8 \mathrm{a}$ & $0,74 \mathrm{~b}$ \\
$15,0^{\circ} \mathrm{C}$ & $22,2 \mathrm{ab}$ & $10,7 \mathrm{a}$ & $12,3 \mathrm{a}$ & $0,64 \mathrm{a}$ \\
\hline
\end{tabular}

* Medias dentro de la misma columna con distinta letra para cada experimento difieren significativamente según la prueba de Tukey $(\mathrm{p}=0,05)$.

$\dagger$ ND: No se determinó.

segundo experimento, las temperaturas de $10,0^{\circ} \mathrm{C}$ y $12,5^{\circ} \mathrm{C}$, presentaron diferencias estadísticas con los demás tratamientos $(\mathrm{p}=0,0131)$.

Para ambos ensayos, se observó una disminución significativa en la firmeza de la pulpa (Cuadro 3) conforme se incrementó la temperatura de almacenamiento $(\mathrm{p}<0,05)$. En los 2 experimentos, el tratamiento control $\left(7,5^{\circ} \mathrm{C}\right)$ presentó la mayor firmeza con valores de 15,2 $\mathrm{N}$ y $12,5 \mathrm{~N}$ respectivamente, mientras que los frutos de piña almacenados en la temperatura de $15,0^{\circ} \mathrm{C}$, presentaron los valores más bajos con $10,9 \mathrm{~N}$ para el primer ensayo, mientras que en el segundo fue de $10,7 \mathrm{~N}$.

El contenido de sólidos solubles y el porcentaje de acidez titulable no se vieron influenciados por las temperaturas a las cuales se almacenaron los frutos (Cuadro 3). Al finalizar los 21+4 dda, se presentaron valores de sólidos solubles entre $11,8 \%$ y $14 \%$ para ambos ensayos; por otro lado, el porcentaje de acidez titulable se encontró entre $0,64 \%$ y $0,78 \%$. Kader (2002) y Garita (2014), indican que el contenido de sólidos solubles mínimo y el porcentaje de acidez titulable máximo para que un fruto de piña sea considerado comerciable debe ser de $12 \%$ y $1 \%$ respectivamente, por lo que los valores obtenidos en este trabajo cumplen con lo establecido para ser considerados comercializables.

Solano (2015) almacenó frutos de piña cv. Dorada Extra Dulce por 21 días a $7,0^{\circ} \mathrm{C}$, y no encontró diferencias significativas para las variables de acidez y porcentaje de sólidos solubles, con valores entre $0,7 \%$ y $0,86 \%$ y $12 \%$ y $13 \%$ respectivamente; asimismo, los valores de firmeza $(\mathrm{N})$ se encontraron entre $10 \mathrm{~N}$ y 20 N. Por otro lado, Hong et ál. (2013), observaron una disminución en el contenido de sólidos solubles en frutos de piña cv. Comte de paris, luego de 24 días de almacenamiento, similar a 
los datos de este trabajo, y lo relacionaron a la utilización de estos como uno de los sustratos de los procesos de respiración, junto a lípidos, proteínas y ácidos orgánicos.

En resumen, los resultados obtenidos sugieren que al aumentar la temperatura de almacenamiento se incrementa el cambio de color y la degradación de clorofila en la cáscara, la tasa respiratoria y la pérdida de peso; además se afecta negativamente la calidad externa de los frutos. Por otro lado, para las variables de calidad interna analizadas, solamente la firmeza de la pulpa disminuyó conforme se incrementó la temperatura de almacenamiento, pues en el tratamiento de $7,5^{\circ} \mathrm{C}$ (control) se presentaron en ambos ensayos, valores de firmeza $(\mathrm{N})$ mayores a los mostrados en los demás tratamientos.

Los datos de este trabajo indican que, la temperatura de almacenamiento podría ser de utilidad en el manejo comercial para incrementar el desarrollo de color amarillo de frutos de piña; sin embargo, debe realizarse más investigación en cuanto a los factores que afectan negativamente la calidad externa de los frutos, especialmente pérdida de peso y desarrollo de moho en el pedúnculo.

\section{AGRADECIMIENTOS}

Los autores de este trabajo agradecen a la empresa Inversiones y Procesadora Tropical S.A. (INPROTSA) y en especial a su gerente de operaciones el Sr. Francisco López Lee, por la colaboración brindada para la realización de este trabajo.

\section{LITERATURA CITADA}

ABDULLAH H., ROHAYA M.A., LATIFAH M.N., SELAMAT M.M., UNDERHILL S. 2002. Respiration rate, ethylene production and chlorophyll content of the fruit and crown of pineapple stored at low temperatures. J. Trop. Agric and Fd Sc. 30(1):99107.

AGRIOS G. 2005. Plant pathology. (5 ed). California, USA, Academic Press. 952 p.

BAERT K., VALERO A., DE MELULENAER B., SAMAPUNDO S., MORSHED M., BO L.,
DEBEVERE J., DEVLIEGHERE F. 2007. Modelling the effect of temperature on the growth rate and lag phase of Penicillium expansum in apples. International Journal of Food Microbiology 118:139-150.

BRAT P., THI HOANG L.N., SOLER A., REYNES M., BRILlOUET J.M. 2004. Physicochemical characterization of a new pineapple hybrid (FLHORAN41 Cv.) Journal of agricultural and food chemistry 52(20):6170-6177.

DERE Ş., GÜNEŞ T., SIVACI R. 1998. Spectrophotometric determination of chlorophyll- A,B and total carotenoids contents of some algae species using different solvents. Turskish Journal of botany 22:13-17.

FAWOLE O.A., OPARA U.L. 2013. Effects of storage temperature and duration on physiological responses of pomegranate fruit. Industrial crops and products 47:300-309.

FONSECA S., OLIVEIRA F., BRECHT J. 2002. Modeling respiration rate of fresh fruits and vegetables for modified atmosphere packages: a review. Journal of food engineering 52:99-119.

GARITA R.A. 2014. La piña. Cartago, Costa Rica, Editorial Tecnológica de Costa Rica. 568 p.

GOUGOULI M., KOUTSOUMANIS K.P. 2010. Modelling growth of Penicillium expansum and Aspergillus niger at constant and fluctuating temperature conditions. International Journal of Food Microbiology 140:252-262.

HASSAN A., OTHMAN Z. 2011. Pineapple (Ananas comosus L. Merr.), pp. 194-217. In: E.M. Yahia (eds.). Postharvest biology and technology of tropical and subtropical fruits. WP.

HONG K., XU H., WANG J., ZHANG L., HU H., JIA Z., GU H., HE Q., GONG D. 2013. Quality changes and internal browning developments of summer pineapple fruit during storage at different temperatures. Scientia Horticulturae 151:68-74.

KADER A. 2002. Postharvest technology of horticultural crops. University of California. Agricultural and natural resources. Third edition. Oakland, California. $535 \mathrm{p}$.

KAYS S. 1997. Postharvest physiology of perishable plant products. Georgia, USA, Exon press. 532 p.

LIU C., LIU Y. 2014. Effects of elevated temperature postharvest on color aspect, physiochemical characteristics, and aroma components of pineapple fruits. Journal of food science 79(12):2409-2414.

MAZUR S.P., NES A., WOLD A.B., REMBERG S.F., MARTINSEN B.K., AABAY K. 2014. Effects of ripeness and cultivar on chemical composition of strawberry (Fragaria $\mathrm{x}$ ananassa Duch.) fruits and their suitability for jam production as a stable product at different storage temperature. Food chemistry 146:412-422. 
PAULL R.E., CHEN C.C. 2003. Postharvest physiology, handling and storage of pineapple, pp. 253-279. In: D.P. Bartholomew, R.E. Paull and K.G. Rohrbach (eds). The pineapple: Botany, production and uses. CABI publishing.

ROBERTS S. 2009. Fruit pigmentation studies. Master of science. Stellenbosch, South Africa, Stellenbosch University. $106 \mathrm{p}$.

SOLANO J. 2015. Efecto del tiempo transcurrido entre cosecha e inicio de enfriamiento en túneles de aire forzado, sobre la vida útil de almacenamiento de piña (Ananas comosus) Var. Dorada Extra Dulce, en dos zonas de Costa Rica. Tesis de licenciatura, Universidad de Costa Rica, San José, Costa Rica. $92 \mathrm{p}$.

SYMONS G.M., CHUA Y.J., ROSS J.J., QUITTENDEN L.J., DAVIES N.W., REID J.B. 2012. Hormonal changes during non-climacteric ripening in strawberry. Journal of experimental botany 63(13):4741-4750.

TIETEL Z., LEWINSOHN E., FALLIK E., PORAT R. 2012. Importance of storage temperatures in maintaining flavor and quality of mandarins. Postharvest biology and technology 64:175-182.

UMAÑA G., LORÍA C.L., GÓMEZ J.C. 2011. Efecto del grado de madurez y las condiciones de almacenamiento sobre las características fisicoquímicas de la papaya híbrido Pococí. Agronomía Costarricense 35(2):61-73.

WANG H.C., HUANG X.M., HU G.B., YANG Z., HUANG H.B. 2005. A comparative study of chlorophyll loss and its related mechanism during Fruit maturation in the pericarp of fast and slow degreening litchi pericarp. Scientia Horticulturae 106:247-257. 